\title{
Persistence of impaired health status of $Q$ fever patients 4 years after the first Dutch outbreak
}

\author{
G. J. M. LIMONARD ${ }^{1 *}$ J. B. PETERS ${ }^{2,3}$, R. BESSELINK ${ }^{4}$, C. A. R. GROOT ${ }^{5}$, \\ P. N. R. DEKHUIJZEN ${ }^{3}$, J. H. VERCOULEN ${ }^{2,3}$ AND \\ M. H. NABUURS-FRANSSEN ${ }^{6}$ \\ ${ }^{1}$ Department of Pulmonary Diseases, Diakonessenhuis, Utrecht, The Netherlands \\ ${ }^{2}$ Department of Medical Psychology, Radboud University Nijmegen Medical Centre, Nijmegen, The Netherlands \\ ${ }^{3}$ Department of Pulmonary Diseases, Radboud University Nijmegen Medical Centre, Nijmegen, The Netherlands \\ ${ }^{4}$ GP Practice Herpen (Huisartsenpraktijk Herpen), Herpen, The Netherlands \\ ${ }^{5}$ Department of Pulmonary Diseases, Bernhoven Hospital, Oss, The Netherlands \\ ${ }^{6}$ Department of Medical Microbiology, Canisius-Wilhelmina Hospital, Nijmegen, The Netherlands
}

Received 15 July 2015; Final revision 27 August 2015; Accepted 1 September 2015;

first published online 28 October 2015

\section{SUMMARY}

A significant proportion of Q fever patients from the first Dutch Q fever outbreak in 2007 showed impairment in health status up to 1 year after infection. Interested in whether this decrease in health status persisted, we set out to determine the health status in the same cohort of patients, 4 years after primary infection and to compare health status scores at the individual patient level between 1 and 4 years follow-up. Health status was assessed with the Nijmegen Clinical Screening Instrument (NCSI). Patients were serologically tested to exclude patients with possible, probable or proven chronic Q fever. Results on the NCSI sub-domains at group level [2008 $(n=54)$ and $2011(n=46)$ ] showed a persistent significant percentage of patients exhibiting clinically relevant ('severe') scores for all NCSI sub-domains. After 4 years, undue fatigue was present in $46 \%$ and exactly half of all patients experienced a severely impaired general quality of life. Patients with NCSI scores available in both 2008 and $2011(n=37)$ showed no difference in all sub-domain scores, except for a small decrease in dyspnoea emotions in 2011. In this group, a significant proportion of patients either improved or worsened in one or more sub-domains of health status. We conclude that at the group level, health status of $\mathrm{Q}$ fever patients remained impaired 4 years after primary infection. At the individual patient level, health status may change.

Key words: Coxiella burnetii, health status, Q fever, quality of life.

\section{INTRODUCTION}

Q fever, a ubiquitous zoonosis caused by the intracellular bacterium Coxiella burnetii, emerged as a human pathogen in The Netherlands in 2007 [1]. Successive

\footnotetext{
* Author for correspondence: G. J. M. Limonard, MD, Diakonessenhuis Utrecht, Department of Pulmonary Diseases, Bosboomstraat 1, 3508TG Utrecht, The Netherlands. (Email: gjmlimonard@gmail.com)
}

annual outbreaks linked to intensified goat farming have amounted to more than 4000 notified and a further estimated 44000 unnotified human cases, the majority of which occurred in the province of Noord-Brabant in the south of The Netherlands [2]. Following extensive governmental measures including restrictions on manure trafficking, vaccination and culling of dairy goats and sheep starting in 2010, the rate of acute human Q fever cases fell markedly. However, since then chronic $\mathrm{Q}$ fever infection has remained a 
significant clinical problem [3]. Accordingly, research efforts have shifted in recent years from management of acute disease to the long-term sequelae of $\mathrm{Q}$ fever; chronic Q fever (vascular infection, endocarditis) and $Q$ fever fatigue syndrome (QFS) [4].

Early reports after the first outbreaks in 2007 and 2008 showed persistent symptomatology, especially fatigue, and a decreased health status of $\mathrm{Q}$ fever patients for a follow-up period of 12-26 months [5, 6]. Health status scores of patients at 12 months vs. 17-26 months followup did not differ significantly, suggesting a long term persistence of impaired health status of equal severity level after 1 year. The original small studies from Australia and England suggest persistent symptomatology and decreased health status can persist for 5-10 years [7, 8].

For the patients of the Dutch Q fever outbreaks, it remains unknown whether impaired health status and symptomatology persist or can change after 1-2 years. In addition to the evaluation of long-term health status at the group level, changes in health status of individual patients have not been studied before.

The first aim of this study was to determine the health status of patients from the first Q fever outbreak in The Netherlands 4 years after primary infection. the second aim was to compare health status scores at the individual patient level between 1 and 4 years follow-up, for those patients for whom data were available at both time points.

\section{METHODS}

\section{Study design and population}

Health status of patients from the 2007 Q fever outbreak was assessed with the Nijmegen Clinical Screening Instrument (NCSI), 4 years after the initial $\mathrm{Q}$ fever infection. The same questionnaire (NCSI) was used in an earlier assessment of health status of patients from the Herpen outbreak cluster at 1 year follow-up [5]. This allows for direct comparisons of scores at the individual patient level between time points of 1 and 4 years follow-up. All surviving patients from the 2007 outbreak followed up in the Herpen GP practices were asked to participate [9]. Patients who did not respond to the initial invitation were contacted by mail and a single telephone call.

\section{Clinical evaluation and questionnaire}

Evaluation of participants included a complete history and physical examination by their own physician, completion of the NCSI questionnaire and a single venous blood sampling for serological testing to exclude patients with possible, probable or proven chronic Q fever. Serological testing consisted of the determination of IFA phase I IgG (Focus Diagnostics, USA) antibodies. Following the diagnostic criteria of the Dutch Q fever Consensus Group, an IgG phase I of $\geqslant 1024$ excluded patients from analysis [10].

\section{Questionnaire: the Nijmegen Clinical Screening Instrument (NCSI)}

The NCSI has been used in previous studies assessing health status after $Q$ fever infection [5, 6, 11-13]. It is based on an empirical definition of health status, covering 'physiological functioning', 'symptoms', 'functional impairment in daily life', and 'quality of life' as main domains. These domains are in turn subdivided into many independent sub-domains. The NCSI is an empirically composed battery of wellvalidated instruments that enable a detailed measurement of these sub-domains of health status [14]. The NCSI provides normative data for each individual sub-domain; increasing scores indicating normal functioning, mild problems or severe problems. We excluded the domain 'physiological function' from the NCSI questionnaire as patients did not perform pulmonary function tests. In this study we measured the same main domains as in 2008; 'symptoms', 'functional impairment' and 'quality of life'. The corresponding sub-domains are 'subjective symptoms', 'dyspnoea emotions', 'fatigue', 'behavioural impairment', 'subjective impairment', 'general quality of life', 'health-related quality of life' and 'satisfaction with relations' [5]. (The sub-domain 'subjective symptoms' represent the patient's overall burden of dyspnoea and experienced dyspnoea during activities. The sub-domain 'dyspnoea emotions' embodies the level of frustration and anxiety a person experiences when dyspnoeic.)

\section{Statistical analysis}

All quantitative data are presented as mea \pm standard error if normally distributed, otherwise median values (with range) are reported. Testing for differences between patients in 2008 and 2011 was performed by Pearson's $\chi^{2}$ or Mann-Whitney test as appropriate. Statistical significance was set at a $P<0.05$. Data were analysed with SPSS v. 14 (SPSS Inc., USA). 


\section{Ethical standards}

All patients provided written informed consent. This study was approved by the local Ethical Board for Human Research (Commissie Mensgebonden Onderzoek file-nr.: 2008/192, ABR nr.: NL 24 404.091·08, amendment dd 3 September 2010).

The authors assert that all procedures contributing to this work comply with the ethical standards of the relevant national and institutional committees on human experimentation and with the Helsinki Declaration of 1975, as revised in 2008 .

\section{RESULTS}

A total of $47(57 \%)$ of 82 surviving Q fever patients from the 2007 outbreak who were followed up in the Herpen GP practices, agreed to participate and were included. All patients underwent clinical evaluation, provided a completed NCSI questionnaire and a blood sample. One of the patients had a phase I IgG of 1024, indicating possible, probable or proven chronic $\mathrm{Q}$ fever, and was referred to an infectious diseases specialist for evaluation, leaving 46 patients for analysis. For 37 patients the NCSI scores were available at both the 1 and 4 years follow-up, allowing for longitudinal comparison of health status at the individual patient level.

In Table 1, patients' characteristics of this study population $(n=46)$ are given for comparison with the data on the patient group assessed in $2008(n=54)$.

Results on the NCSI sub-domains at the group level (Table 2) for $2008(n=54)$ and $2011(n=46)$ show a persistent significant percentage of patients exhibiting clinically relevant ('severe') scores for all NCSI subdomains. No significant differences were found between patients with or without comorbidities on any of the NCSI sub-domains. Overall, severe subjective symptoms were present in $35 \%$ of patients. After 4 years, undue fatigue was present in $46 \%(52 \%$ after 1 year) and exactly half of all patients experienced a severely impaired general quality of life.

Comparison of sub-domains for patients with NCSI scores available for both 2008 and $2011 \quad(n=37$, Table 3) showed no difference in all sub-domain scores, except for a small but statistically significant decrease in dyspnoea emotions.

Table 4 shows the dynamics of NCSI scores at the individual patient level, as percentages of patients whose scores either improved, remained equal or worsened between 2008 and 2011. A significant proportion
Table 1. Patients' characteristics

\begin{tabular}{lll}
\hline \hline & $\begin{array}{l}2008^{*} \\
(N=54) \\
n(\%)\end{array}$ & $\begin{array}{l}2011 \\
(N=46) \\
n(\%)\end{array}$ \\
\hline Male & $33(61)$ & $28(61)$ \\
Age, yr, mean (s.D.) & $53(14)$ & $56(14)$ \\
$\quad$ Range, yr & $20-81$ & $23-84$ \\
Comorbidity & $22(41)$ & $22(48)$ \\
Smoking status & & \\
$\quad$ Current & $24(44)$ & $21(46)$ \\
Former & $19(35)$ & $15(33)$ \\
Never & $11(20)$ & $10(22)$ \\
\hline
\end{tabular}

Values given are $n(\%)$ unless stated otherwise.

* See reference [5].

of all individual Q fever patients either improved or worsened in one or more sub-domains of health status. Fatigue scores improved in about one-third of patients, but worsened in one in five $(21 \%)$. More than $30 \%$ of patients showed significant improvement in the sub-domains of 'fatigue', 'subjective impairment' and 'health-related quality of life', but these improvements were cancelled out at the group level by patients with worsening scores.

\section{DISCUSSION}

Persistence of impairment in many sub-domains of health status after 4 years in the majority of affected $\mathrm{Q}$ fever patients is marked, and suggests an absence of further dynamics in symptomatology after 1 year. These findings tally with earlier research in England, Australia and Canada on very long-term sequelae post-Q fever infection, reporting increased symptomatology including similar levels of fatigue up to 10 years after primary infection [15-17]. A further finding is that at the individual patient level, although the majority remain equally impaired, patients can improve or worsen on an individual basis in one or more sub-domains of health status between 1 and 4 years.

This study has several strengths and weaknesses that need to be addressed. This is the first report on longitudinal long-term health status of $Q$ fever patients in The Netherlands with more than 2 years follow-up. The availability of detailed health status data at follow-up points 1 year and 4 years for the same patients allowed comparison at the individual patient level, a feature that has not been studied before. Moreover, serology was performed to exclude chronic disease. As the background prevalence of 
Table 2. Percentage of patients with normal, elevated or severe scores on the NCSI in 2008 and 2011

\begin{tabular}{|c|c|c|c|c|c|c|}
\hline \multirow[b]{2}{*}{ Main domain/sub-domain } & \multicolumn{3}{|c|}{$2008(N=54)$} & \multicolumn{3}{|c|}{$2011(N=46)$} \\
\hline & Normal & Elevated & Severe & Normal & Elevated & Severe \\
\hline \multicolumn{7}{|l|}{ Symptoms } \\
\hline Subjective symptoms & 22 & 44 & 33 & 20 & 46 & 35 \\
\hline Dyspnoea emotions & 41 & 17 & 43 & 41 & 22 & 37 \\
\hline Fatigue & 26 & 22 & 52 & 33 & 22 & 46 \\
\hline \multicolumn{7}{|l|}{ Functional impairment } \\
\hline Behavioural impairment & 48 & 35 & 17 & 46 & 33 & 22 \\
\hline Subjective impairment & 19 & 54 & 28 & 28 & 41 & 30 \\
\hline \multicolumn{7}{|l|}{ Quality of life (QoL) } \\
\hline General QoL & 41 & - & 59 & 50 & - & 50 \\
\hline Health-related QoL & 46 & 30 & 24 & 50 & 22 & 28 \\
\hline Satisfaction with relations & 37 & 39 & 24 & 59 & 24 & 17 \\
\hline
\end{tabular}

NCSI, Nijmegen Clinical Screening Instrument.

All values are percentages.

Table 3. Comparison of NCSI scores for all sub-domains* at the individual patient level between 2008 and $2011(N=37)$

\begin{tabular}{|c|c|c|c|c|c|}
\hline \multirow[b]{2}{*}{ Main domain/sub-domain } & \multicolumn{2}{|l|}{2008} & \multicolumn{2}{|l|}{2011} & \multirow[b]{2}{*}{ Sig. } \\
\hline & Mean & (s.D.) & Mean & (s.D.) & \\
\hline \multicolumn{6}{|l|}{ Symptoms } \\
\hline Subjective symptoms & 6.9 & $(4 \cdot 9)$ & $6 \cdot 9$ & $(5 \cdot 5)$ & 0.97 n.s. \\
\hline Dyspnoea emotions & $10 \cdot 1$ & $(4 \cdot 5)$ & 8.7 & $(3 \cdot 9)$ & $0 \cdot 01$ \\
\hline Fatigue & $33 \cdot 4$ & $(15 \cdot 1)$ & $31 \cdot 5$ & $(13 \cdot 1)$ & 0.26 n.s. \\
\hline \multicolumn{6}{|l|}{ Functional impairment } \\
\hline Behavioural impairment & $8 \cdot 7$ & $(12 \cdot 6)$ & $9 \cdot 5$ & $(13 \cdot 9)$ & 0.63 n.s. \\
\hline Subjective impairment & $9 \cdot 8$ & $(5 \cdot 7)$ & $9 \cdot 2$ & $(5 \cdot 2)$ & 0.46 n.s. \\
\hline \multicolumn{6}{|l|}{ Quality of life (QoL) } \\
\hline General QoL & $20 \cdot 1$ & $(19 \cdot 5)$ & $18 \cdot 3$ & $(19 \cdot 15)$ & 0.54 n.s. \\
\hline Health-related QoL & $4 \cdot 0$ & $(1 \cdot 9)$ & $4 \cdot 0$ & $(2 \cdot 1)$ & 1.00 n.s. \\
\hline Satisfaction with relations & $3 \cdot 5$ & $(1 \cdot 8)$ & $3 \cdot 4$ & $(2 \cdot 2)$ & 0.71 n.s. \\
\hline
\end{tabular}

NCSI, Nijmegen Clinical Screening Instrument; n.s., not significant.

* The higher the score, the more problematic, for reference values for all sub-domains see reference [14].

serological evidence of past Q fever infection in endemic regions in The Netherlands reaches up to $15 \%$ and chronic Q fever can develop in $1-5 \%$ of $\mathrm{Q}$ fever patients, this is highly relevant [18].

The limited patient numbers in this study is a weakness, and precludes generalization of the obtained results to the entire Dutch Q fever outbreak population. The small patient numbers are largely due to the fact that the study cohort comprised the very first, local group of Q fever patients in The Netherlands from the rural town of Herpen, in the province of Noord-Brabant [9]. Furthermore, the absence of a proper Q fever negative control group at the 2011 time point does not allow for an estimation of how much impairment of health status is actually directly caused by the primary infection with $C$. burnetii.

This study provides further evidence for persistent symptomatology and long-term decreased health status of Q fever patients in The Netherlands. Research groups in England, Australia and Canada have similarly found consistent and clearly decreased long-term health status outcomes in Q fever patients, using various/different questionnaires and study methods [15-17]. The acknowledgement and acceptance of a protracted fatigued state following acute Q fever in about $20 \%$ of patients, sometimes designated post-Q fever fatigue syndrome (PQFS); however, is not universal $[19,20]$. The main reasons for this reluctance 
Table 4. Percentages of patients whose NCSI scores improved, remained equal or worsened* at the individual patient level, between follow-up time points 2008 and $2011(N=37)$.

\begin{tabular}{llll}
\hline \hline Main domain/sub-domain & Improved & Equal & Worsened \\
\hline Symptoms & & & \\
$\quad$ Subjective symptoms & 24 & 58 & 18 \\
$\quad$ Dyspnoea emotions & 29 & 66 & 5 \\
$\quad$ Fatigue & 34 & 45 & 2 \\
Functional impairment & & & \\
$\quad$ Behavioural impairment & 5 & 82 & 13 \\
$\quad$ Subjective impairment & 32 & 50 & 18 \\
Quality of life & & & \\
$\quad$ General QoL & 26 & 55 & 18 \\
$\quad$ HRQoL & 37 & 32 & 32 \\
$\quad$ Satisfaction with relations & 21 & 68 & 11 \\
\hline
\end{tabular}

* Differences between scores in 2008 and 2011 were calculated as follows: (score 2008 - score 2011)/s.D. 2008.

A difference on a sub-domain score of $<-0.5$ corresponds with significant improvement; a score of $>-0.5$ and $<0.5$ means no significant change and a score of $>0.5$ corresponds to worsening of health status in that sub-domain.

All values are percentages.

seem to be the incompletely understood pathophysiology of PQFS, the absence of a diagnostic test and possibly, fear of patients' financial compensation claims. Moreover, post-infectious protracted fatigue states have been reported for several other bacterial and viral pathogens such as Bartonella, Legionella, Epstein-Barr virus, cytomegalovirus and West Nile virus [21, 22]. A recent Dutch study, using the NCSI and the Short Form 36 (SF-36), compared the health status of 309 Q fever patients and 190 patients with Legionnaires' disease and found severe impairment of various health status sub-domains including fatigue for both diseases 1 year after onset of illness [11]. Another smaller study from The Netherlands compared 50 patients with a lower respiratory tract infection (LRTI) caused by Q fever with 32 non-Q fever LRTI patients with a follow-up period of 10-19 months. With the exception of the sub-domain 'subjective pulmonary symptoms', no difference was observed in health status for all other measured subdomains using the NCSI [12]. Interestingly, as in our study, significant proportions of $\mathrm{Q}$ fever patients experienced severe fatigue $(40 \%)$ and severely impaired general quality of life (40\%).

There are numerous studies probing the underlying pathophysiology of PQFS that show significant differences between $\mathrm{Q}$ fever patients with and without
PQFS with regard to gene expression [23-25], serum cytokine levels [26] and peripheral blood mononuclear cells cytokine release patterns after stimulation with C. burnetii antigens [26, 27]. However, there are several shortcomings that impede a thorough interpretation and unifying concept of PQFS pathophysioloy; a lack of a clear definition of PQFS, small patient numbers and different laboratory techniques and protocols that do not allow for ready comparison and validation of obtained results. Recently, a new unifying paradigm has been put forward for PQFS, postulating PQFS symptomatology to be underpinned by the interaction of persistent Coxiella specific non-viable cell residues with immunogenetic polymorphisms in the host $[28,29]$.

Moreover, there is no validated evidence-based treatment for PQFS. To address this last issue, a large randomized placebo-controlled prospective clinical trial is underway in The Netherlands assessing the efficacy of long-term treatment with doxycycline and cognitive behavioural therapy in PQFS patients [30].

In conclusion, these data support an overall sustained impaired health status of Q fever patients in The Netherlands 4 years after primary infection. A minority of Q fever patients with impaired health status in 2008 showed improvement in various subdomains of health status by 2011 , but at the group level this effect was cancelled out by patients with worsening scores.

\section{ACKNOWLEDGEMENTS}

This research received no specific grant from any funding agency, commercial or not-for-profit sectors.

\section{DECLARATION OF INTEREST}

None.

\section{REFERENCES}

1. Van Steenbergen JE, et al. An outbreak of Q fever in The Netherlands - possible link to goats [in Dutch]. Nederlands Tijdschrift voor Geneeskunde 2007; 151: 1998-2003.

2. Van der Hoek W, et al. Relation between Q fever notifications and Coxiella burnetii infections during the 2009 outbreak in The Netherlands. Eurosurveillance 2012; 19; 20058.

3. Schneeberger PM, et al. Q fever in the Netherlands 2007-2010: what we learned from the largest outbreak ever. Médecine et Maladies Infectieuses 2014; 44: 339 353. 
4. Van der Hoek W, et al. Shifting priorities in the aftermath of a Q fever epidemic in 2007 to 2009 in The Netherlands: from acute to chronic infection. Eurosurveillance 2012; 19: 20059.

5. Limonard GJ, et al. Detailed analysis of health status of Q fever patients 1 year after the first Dutch outbreak: a case-control study. QJM: An International Journal of Medicine 2010; 103: 953-958.

6. Morroy G, et al. The health status of Q-fever patients after long-term follow-up. BMC Infectious Diseases 2011; 18: 97.

7. Marmion BP, et al. Protracted debility and fatigue after acute Q fever. Lancet 1996; 347: 977-978

8. Ayres JG, Smith EG, Flint N. Protracted fatigue and debility after acute Q fever. Lancet 1996; 347: 978-979.

9. Limonard GJ, et al. One-year follow-up of patients of the ongoing Dutch Q fever outbreak: clinical, serological and echocardiographic findings. Infection 2010; 38: 471-477.

10. Wegdam-Blans MC, et al. Chronic Q fever: review of the literature and a proposal of new diagnostic criteria. Journal of Infection 2012; 64: 247-259.

11. Van Loenhout JA, et al. Serious long-term health consequences of Q-fever and Legionnaires' disease. Journal of Infection 2014; 68: 527-533.

12. Van Dam AS, et al. A cross-sectional study to assess the long-term health status of patients with lower respiratory tract infections including $\mathrm{Q}$ fever. Epidemiology and Infection 2015; 143: 48-54.

13. Kremers MN, et al. Correlations between peripheral blood Coxiella burnetii DNA load, interleukin-6 levels, and $\mathrm{C}$-reactive protein levels in patients with acute $\mathrm{Q}$ fever. Clinical and Vaccine Immunology 2014; 21: 484487.

14. Peters JB, et al. Development of a battery of instruments for detailed measurement of health status in patients with COPD in routine care: the Nijmegen Clinical Screening Instrument. Quality of Life Research 2009; 18: 901-912.

15. Ayres JG, et al. Post-infection fatigue syndrome following Q fever. QJM: An International Journal of Medicine 1998; 91: 105-123.

16. Wildman MJ, et al. Chronic fatigue following infection by Coxiella burnetii ( $\mathrm{Q}$ fever): ten-year follow-up of the 1989 UK outbreak cohort. QJM: An International Journal of Medicine 2002; 95: 527-538.

17. Hatchette TF, et al. The effect of $C$. burnetii infection on the quality of life of patients following an outbreak of Q fever. Epidemiology and Infection 2003; 130: 491-495.
18. Van der Hoek W, et al. Epidemic Q fever in humans in the Netherlands. Advances in Experimental Medicine and Biology 2012; 984: 329-364.

19. Raoult D. Q fever: still a mysterious disease. QJM: $A n$ International Journal of Medicine 2002; 95: 491-492.

20. Strauss B, et al. Are fatigue symptoms and chronic fatigue syndrome following $\mathrm{Q}$ fever infection related to psychosocial variables? Journal of Psychosomatic Research 2012; 72: 300-304.

21. Hickie I, et al. Post-infective and chronic fatigue syndromes precipitated by viral and non-viral pathogens: prospective cohort study. British Medical Journal 2006; 16; 333.

22. Garcia MN, et al. Evaluation of prolonged fatigue post-West Nile virus infection and association of fatigue with elevated antiviral and proinflammatory cytokines. Viral Immunology 2014; 27: 327-333.

23. Kerr JR, et al. Gene expression subtypes in patients with chronic fatigue syndrome/myalgic encephalomyelitis. Journal of Infectious Diseases 2008; 15; 197: 1171-1184.

24. Helbig $\mathbf{K}$, et al. Immune response genes in the post-Q-fever fatigue syndrome, Q fever endocarditis and uncomplicated acute primary $\mathrm{Q}$ fever. QJM: $A n$ International Journal of Medicine 2005; 98: 565-574.

25. Piraino B, Vollmer-Conna U, Lloyd AR. Genetic associations of fatigue and other symptom domains of the acute sickness response to infection. Brain, Behaviour and Immunity 2012; 26: 552-558.

26. Penttila IA, et al. Cytokine dysregulation in the post-Q-fever fatigue syndrome. QJM: An International Journal of Medicine 1998; 91: 549-560.

27. Vollmer-Conna U, et al. Cytokine polymorphisms have a synergistic effect on severity of the acute sickness response to infection. Clinical Infectious Diseases 2008; 47: 1418-1425.

28. Marmion BP, et al. Q fever: persistence of antigenic non-viable cell residues of Coxiella burnetii in the host-implications for post $\mathrm{Q}$ fever infection fatigue syndrome and other chronic sequelae. QJM: $A n$ International Journal of Medicine 2009; 102: 673-684.

29. Sukocheva OA, et al. Long-term persistence after acute $\mathrm{Q}$ fever of non-infective Coxiella burnetii cell components, including antigens. QJM: An International Journal of Medicine 2010; 103: 847-863.

30. Keijmel SP, et al. The Qure study: Q fever fatigue syndrome - response to treatment; a randomized placebocontrolled trial. BMC Infectious Diseases 2013; 27; 157. 